\title{
ENSEÑANZA DE LA ESCRITURA EN EL ÁMBITO UNIVERSITARIO: SITUACIÓN ACTUAL Y PERSPECTIVAS
}

https://doi.org/10.7203/Normas.1.4646

\author{
TEACHING OF THE WRITING IN THE UNIVERSITY AREA: CURRENT SITUATION \\ AND PERSPECTIVES
}

Eduardo ESPAÑA PALOP

Universidad Católica de Valencia, «San Vicente Mártir»

RESUMEN:

En el presente artículo se plantea, a través del análisis de textos producidos por alumnos universitarios, si realmente estos alumnos poseen las destrezas suficientes para considerar que pueden alcanzar un nivel $\mathrm{C} 1$ de español al finalizar sus estudios. El autor analiza cuáles son los principales errores que cometen estos alumnos en sus producciones textuales y se pregunta cuál es la función que los docentes universitarios deben desempeñar en la enseñanza de la composición de textos escritos.

PALABRAS CLAVE: análisis, textos escritos, nivel C1, español.

\section{ABSTRACT:}

This paper argues, through the analysis of texts produced by university students, if really these students have the skills to consider that they can reach a C1 level of Spanish when they will finish their university studies. The author analyzes which are the principal mistakes that these students commit in their textual productions and wonders about which is the function that university teachers must have in the education of written texts

KEY WORDS: analysis, written texts, C1 level, Spanish.

\section{INTRODUCCIÓN}

Con la introducción de los nuevos planes de estudio en el ámbito universitario, uno de los objetivos que deben alcanzar los alumnos universitarios es el nivel $\mathrm{C} 1$ en español. En muchas ocasiones se da por supuesto que siendo alumnos cuya lengua materna es el español y con sus estudios cursados en español esto debería ser así. 
Recordemos que el nivel $\mathrm{C} 1$ de una lengua supone que el alumno puede hacer un uso flexible y efectivo del idioma para fines sociales, académicos y profesionales y que puede producir textos claros, bien estructurados y detallados sobre temas de cierta complejidad, mostrando un uso correcto de los mecanismos de organización, articulación y cohesión del texto. Es decir, el alumno universitario debería ser capaz de escribir sobre temas académicos usando un lenguaje apropiado y realizando textos correctos desde todos los puntos de vista.

A lo largo de su vida académica, el alumno se debe enfrentar a dos tipos de composiciones escritas básicas: el examen y el trabajo académico. Ambos tipos de composiciones comparten muchos rasgos en común, pues ambos se tratan, a fin de cuentas, de textos escritos que requieren una estructuración concreta y el uso de un lenguaje académico. Sin embargo hay ciertas características que los diferencian. Las dos diferencias principales son: por un lado, el trabajo académico posee una superestructura que el examen no posee. Es decir, el trabajo académico, más allá de su nivel textual, debe seguir una ordenación y una estructuración de sus diferentes partes. Además, el hecho de que esté compuesto por diferentes partes, hace que dentro del trabajo académico podamos encontrar diversos tipos de textos, desde los textos expositivos pasando por los argumentativos o enumerativos. La segunda de las diferencias es que el trabajo académico es un trabajo dilatado en el tiempo y sujeto a una o varias revisiones, tanto por la persona que lo realiza como por otras personas. Mientras que el texto de un examen debe ser realizado en un momento concreto y solamente puede ser revisado por la persona que lo realiza.

Es por estas razones por las que, dentro de las dos posibilidades que nos ofrecen los textos académicos, nos hemos propuesto analizar las composiciones derivadas de la realización de exámenes por parte de los alumnos. Creemos que pueden ser más significativas del nivel de dominio de la escritura real que poseen, pues son textos en los que no pueden acudir a un revisor externo y, además, se deben realizar en el momento, sin posibilidad de que el alumno pueda acogerse a ningún tipo de corrector informático o manual gramatical que le sirvan de apoyo.

A su vez, los textos resultado de la realización de un examen pueden ser de diversos tipos. Los dos tipos de textos más comunes que nos podemos encontrar son los textos expositivos y los argumentativos. Ambos tipos de textos, pese a sus diferencias, comparten unas mismas bases textuales en cuanto a la coherencia, cohesión y adecuación.

Para la realización de este estudio, vamos a basarnos en los exámenes de dos asignaturas distintas del grado de magisterio. Una de las asignaturas es «Lengua española para maestros». Esta es una asignatura obligatoria del primer curso tanto para el grado de infantil como para el grado de primaria. El examen de esta asignatura consta de dos partes, una primera parte teórico-práctica en la que el alumno debe responder una serie de preguntas relacionadas con la norma del español actual y una segunda parte totalmente práctica en la que el alumno debe realizar un texto argumentativo en torno a una serie de temas de actualidad que se le proporcionan. La segunda de las asignaturas 
es "Adquisición y desarrollo del lenguaje oral infantil". Es una asignatura de segundo curso dentro del grado de infantil. En esta asignatura el examen constaba de una primera parte tipo test y una segunda parte consistente en una pregunta a la que el alumno debía dar una respuesta de forma desarrollada (el espacio del que disponía era de un folio por ambas caras). Todos los alumnos de esta asignatura habían cursado previamente la anterior asignatura.

Partiendo de estas dos asignaturas, en este artículo nos proponemos realizar un análisis de los principales errores cometidos por alumnos que han cursado ambas asignaturas en este curso 2010-2011. El objetivo que nos planteamos es doble, por un lado, observar cuáles son los principales errores que cometen los alumnos a la hora de realizar los textos. El segundo de los objetivos es comprobar si existe algún tipo de evolución entre los alumnos de primer año y los alumnos de segundo año. Los errores los vamos a dividir en cuatro grandes secciones: errores de coherencia, de cohesión, de adecuación y errores ortográficos.

\section{LENGUA ESPAÑOLA PARA MAESTROS}

Esta asignatura consta de cuarenta y cinco alumnos matriculados, de los cuales cuarenta y uno se presentaron en una primera convocatoria y cuatro en la segunda convocatoria. Para la realización del texto, el alumno contaba con una hora y media de tiempo y con el espacio de un folio por ambas caras.

\subsection{Errores de coherencia}

De los cuarenta y cinco textos estudiados, cuarenta de ellos presentan una organización externa basada en introducción, nudo y desenlace. De estos cuarenta textos, solo en quince de ellos observamos que en la conclusión se retoman las ideas aportadas en la introducción. En el resto de los textos se realiza una introducción general sobre el tema y la conclusión es donde el alumno expresa su opinión personal. De los cinco textos restantes, cuatro están escritos sin una introducción, pero sí con una conclusión y al texto restante le falta la conclusión.

En cuanto a la coherencia interna del texto, los alumnos presentan problemas, sobre todo en lo que se refiere a la creación y organización de los párrafos. De los cuarenta y cinco textos, únicamente en cinco encontramos que la totalidad de los párrafos se encuentran bien construidos. En el resto de los textos hay errores en mayor o menor medida en lo que se refiere a la construcción de los párrafos. 
Uno de los errores más comunes es la construcción de párrafos demasiado cortos o párrafos demasiado largos. Encontramos que muchos textos contienen párrafos como los siguientes, construidos con una única oración:

1. El estándar es la variedad de lengua que puede ser entendida en todos los países de habla hispana.

2. Finalmente, concluimos diciendo que todas las variantes del español son igual de importantes y no hay una mejor que otra.

También encontramos el caso contrario, párrafos excesivamente largos que podrían subdividirse en varios párrafos de menor tamaño:

3. Ninguna de las variedades del español es mejor que otra, todas las variedades son iguales. En todos los países se habla un dialecto que es igualmente respetable, aunque todos sean distintos. Lo que une todos esos dialectos y el español es el estándar. En Venezuela encontramos un dialecto propio del español. Este dialecto de Venezuela tiene diversos niveles, como todos los dialectos del español. En Venezuela también hay nivel culto, estándar e informal. Si decimos que una variedad es mejor que otra, entramos en racismo lingüístico. Debemos tener claro que ninguna variedad es mejor. Simplemente son diferentes. Asimismo, en España también se hablan variedades distintas y todas son igualmente aceptadas. No podemos decir que los dialectos de los países de América son peores cuando en España tenemos dialectos muy diferentes. Además, muchos dialectos de España son parecidos a los dialectos de los países de América.

De este párrafo fácilmente podríamos extraer tres párrafos distintos. Un primer párrafo que serían las tres primeras oraciones. Un segundo párrafo serían las seis oraciones siguientes. Finalmente, tendríamos un tercer párrafo con el resto de las oraciones que faltan.

Otro de los errores comunes que encontramos en la construcción de párrafos está relacionado con la progresión temática dentro de los mismos. En los textos aparecen párrafos en los que se engloban informaciones muy diferentes:

4. En mi opinión, sí que hay variantes correctas e incorrectas del Español. Pero estas variantes no están relacionadas con las zonas geográficas. Las variantes incorrectas del español son las que habla la gente de la calle que no tiene estudios ni se preocupa de la corrección del lenguaje. En Venezuela no sé si pasa lo mismo. Como el texto dice en Venezuela hay diversas variantes geográficas. Esas variantes son igual de respetables que las variantes de España. En España tenemos también diversos dialectos $(\ldots)$. 
En este párrafo, el alumno empieza tratando las distintas variantes del español según el nivel de estudios de la persona o según sea una situación formal o informal. De ahí evoluciona a que en Venezuela hay diversas variantes geográficas que son igual de respetables que las españolas. Y, para finalizar, empieza a explicar los diversos dialectos que podemos encontrar en España.

También encontramos párrafos distintos que contienen informaciones, o repetidas en otro párrafo, o informaciones que están en párrafos distintos y que deberían estar contenidas en un mismo párrafo:

5. En el artículo que se nos proporciona se destaca la visión negativa que tienen los propios hablantes del dialecto cuando lo ponen en práctica. Esta visión negativa coincide con las posturas tradicionales con respecto a las variantes dialectales. (...)

(...) La postura negativa de los hablantes de los dialectos muestra que los prejuicios tradicionales no se han superado. Estos prejuicios tradicionales vienen influidos por ciertas visiones lingüísticas (...).

\subsection{Errores de cohesión}

Todos los textos presentaban algún tipo de error de cohesión. Los errores de cohesión más comunes, por este orden, han sido: en primer lugar, un mal uso de los signos de puntuación. Como ejemplos, sirvan los siguientes:

6. Como es explicado en el artículo precedente los hablantes de este dialecto son nativos de Venezuela.

7. Los habitantes de esta zona de sudamérica en su mayoría nativos, son los últimos que conservan este dialecto especial.

En estas dos oraciones hay un mal uso de la coma. En la número seis, el alumnos no usa la coma para separar el inciso que aparece al principio de la oración principal. Y en la número siete, el alumno no escribe la coma que debería ir delante del inciso explicativo que acompaña al sujeto de la oración.

Otro error muy común en la mayoría de los estudiantes es el hecho de que construyen oraciones demasiado largas. Esto da lugar a dos fenómenos: el primero de ellos es que algunas veces, es demasiado complicado entender la oración que se está leyendo, y el lector pierde el sentido de lo que se quiere expresar:

8. Como ejemplo podemos poner que en ciertas partes de España, algunos de los habitantes también hablan unos dialectos que no corresponden con el estándar 
habitual, sino que son dialectos propios de esa zona que contienen sus variantes particulares y esto hace que otras personas tengan dificultades cuando tienen que entenderlos.

Esta oración la podríamos subdividir en diversas oraciones que facilitarían la comprensión de la información:

9. Como ejemplo podemos poner que en ciertas partes de España, algunos de los habitantes también hablan unos dialectos que no corresponden con el estándar habitual. Estos dialectos contienen variantes particulares. Esto hace que otras personas tengan dificultades cuando tienen que entenderlos.

Otro error derivado del hecho de construir oraciones demasiado largas consiste en que algunas veces hay fallos de concordancia debido al exceso de oraciones subordinadas que aparecen en el interior de estas oraciones:

10. Todas la gente, sea del país que sea, que habla español de manera habitual y como lenguaje materno en su día a día usan un dialecto propio.

En esta oración se produce una mala concordancia de número entre el sujeto y el núcleo del predicado. Lo que sucede es que entre el sujeto y el predicad hay demasiados elementos, esto hace que el alumno concuerde no con la palabra en sí, sino con la idea de esa palabra. Al no tener la palabra cerca, se produce una concordancia ad sensum.

También es bastante habitual encontrar un uso inadecuado de los marcadores del discurso. Normalmente este uso inadecuado consiste en atribuir significados que no posee a un marcador discursivo, como por ejemplo:

11. Todos las distintas variantes del español pueden ser englobadas dentro de un dialecto u otro, en consecuencia, el estándar es la variedad neutra que no puede ser adscrita a ningún dialecto.

Según el Diccionario de partículas discursivas del español, el uso de en consecuencia es: «presenta el miembro del discurso como efecto o consecuencia objetiva del miembro del discurso anterior». Por tanto, se puede comprobar que en este contexto no está siendo utilizado correctamente. 


\subsection{Errores de adecuación}

El error de adecuación más común que encontramos en los textos analizados es el uso de palabras y expresiones propias del ámbito informal coloquial en el texto escrito. Podemos observar este fenómeno en fragmentos como los siguientes:

12. Porque aunque no sea muy correcto que los amigos hablen con otros colegas en ese dialecto, esto suele pasar porque no conocen otra cosa.

13. Cuando la gente va a dar una vuelta por ahí se encuentra que la mayoría de la peña habla igual que ellos.

En los ejemplos doce y trece se utilizan los términos colegas, peña y dar una vuelta por ahí, que son propios de un ámbito coloquial informal y que no deberían aparecer en un texto escrito propio del ámbito formal.

Otro error relacionado también con el ámbito informal coloquial es el uso de palabras comodín o de palabras con significados vagos e imprecisos:

14. Todas estas visiones negativas de la lengua son cosas que van quedando en el imaginario colectivo y hacen que otras personas tengan cosas en contra de la lengua.

En una misma oración el alumno usa dos veces el término cosas, término muy vago y que debe ser evitado en un lenguaje formal debido a su imprecisión. Sería conveniente sustituir este término por otros más exactos. Dos posibles soluciones podrían ser la palabras prejuicios y sentimientos negativos.

\subsection{Errores ortográficos}

La mayoría de los textos que han sido analizados no presentan errores ortográficos. El error ortográfico más común es la omisión de la tilde, presente en mayor o menor medida en veinticinco de los textos estudiados:

15. En Venezuela habia antes muchas mas personas que hablaban esta variante dialectal.

16. El estandar es la variante que unifica todos los dialectos del español, sin importar la region de donde procedan. 
Observamos en la primera oración dos errores típicos de acentuación, la falta de tilde para romper el diptongo en había y la falta de tilde diacrítica en más. En la segunda oración falta la tilde en estándar y en región.

En cuanto a errores ortográficos consistentes en la escritura errónea de ciertas palabras, estos pueden deberse a dos factores. El primero de ellos, la interferencia entre el castellano y el valenciano, lo que hace que ciertas palabras se escriban con la grafía que les corresponde en valenciano, error este presente únicamente en cinco de los textos:

17. En los estudios sobre la cuestion no havian muchos datos que clarificasen las desviaciones sobre la norma producidas.

En valenciano el verbo haber se escribe con $v$. Este es uno de los errores por interferencia más comunes entre los hablantes bilingües de castellano y valenciano (además de la concordancia del impersonal haber, general entre hablantes monolingües y bilingües).

El segundo de los factores es, simplemente, la confusión del alumno en la escritura de alguna de las grafías problemáticas del español. Este error lo podemos encontrar en diez de los textos analizados:

18. Algunos de los estudios sobre el estándar han echo más mal que bien al concepto de estándar que $a$ tenido la mayoría de la población a lo largo de estos últimos años.

\section{ADQUISICIÓN Y DESARROLLO DEL LENGUAJE ORAL INFANTIL}

En esta asignatura había cuarenta y dos alumnos matriculados. En la primera convocatoria se presentaron treinta y ocho alumnos y en la segunda convocatoria se presentaron nueve alumnos, pues varios alumnos suspendieron la primera convocatoria y se tuvieron que volver a examinar.

\subsection{Errores de coherencia}

La gran mayoría de los textos corregidos, treinta y cinco, no tienen ninguna estructura externa reconocible. El alumno se limita a exponer los conocimientos sin dotar al texto de ningún tipo de introducción o conclusión. En los textos restantes encontramos en diez de ellos que el texto sí que posee introducción pero no conclusión; 
y finalmente, únicamente en dos de los textos encontramos una introducción y una conclusión.

En cuanto a la coherencia interna del texto, volvemos a encontrar problemas en la construcción de párrafos. De los cuarenta y nueve textos, únicamente quince no tienen ningún problema en lo que a la formación de párrafos se refiere. El resto de los textos tiene algún tipo de problemas. Estos errores, al igual que en los casos anteriores, consisten en la construcción de párrafos demasiado largos (que deberían constituir diversos párrafos independientes) o párrafos demasiado cortos (insuficiente entidad para ser considerado un párrafo independiente), como podemos observar en los ejemplos siguientes:

19. Un niño de tres años, en el plano fonológico, domina la mayoría de los fonemas de la lengua materna.

20. La gramática es la parte de la lingüística que se ocupa del estudio del código o sistema lingüístico y se divide en fonética y fonología, morfología, sintaxis y pragmática. Esto último se encarga del estudio del plano textual de la lengua y se divide en dos niveles: comunicativo y enunciativo. En el plano comunicativo nos encontramos como componentes el texto y el discurso. Los componentes del plano enunciativo son la oración y el enunciado. Podemos definir oración como la construcción mínima con la que el hablante enuncia algo. El enunciado es el esquema, la forma en que se relacionan las oraciones sin que ninguno sea nuclear con respecto a los demás. La diferencia funtadmental entre texto y discurso es la relación paradigmática del texto frente a la sintagmática del discurso.

En el ejemplo número veinte sería más aconsejable dividir el párrafo en, como mínimo, dos párrafos distintos. El primero de ellos estaría constituido por las tres primeras oraciones y el segundo párrafo quedaría formado por las cuatro oraciones restantes.

También encontramos errores como en los ejemplos anteriores relacionados con la progresión temática de los párrafos. En estos textos, encontramos que en un mismo párrafo aparecen informaciones muy diferentes y que sería mejor que apareciesen en párrafos distintos, como por ejemplo:

21. La competencia lingüística es el conjunto de conocimientos y habilidades que el hablante debe adquirir para poder conseguir una buena comunicación en una situación concreta. También es el contenido para poder alcanzar el éxito en una situación lingüística de comunicación. Mientras que la competencia comunicativa no se aprende. Se adquiere, pues a hablar se aprende hablando. La adquisición del lenguaje es inconsciente y el aprendizaje es consciente. En la competencia comunicativa está la interlengua, que es un proceso intermedio porque nunca se termina de aprender. Siempre estamos en el proceso intermedio. 
Podemos comprobar que en este párrafo se trata sobre tres conceptos muy distintos que el alumno no relaciona entre sí, sino que los aúna todos juntos en el párrafo. Los tres conceptos que el alumno une son competencia comunicativa, competencia lingüística e interlengua.

En estos textos no encontramos, a diferencia de los estudiados en el apartado anterior, informaciones que se repitan de un párrafo a otro. Posiblemente, esto venga dado por le hecho de que son exámenes más teóricos y el alumno repite menos informaciones.

\subsection{Errores de cohesión}

Volvemos a encontrar en estos textos la característica de que todos ellos presentaban algún tipo de error de cohesión. A su vez, también en estos textos, los errores más habituales eran los errores relacionados con los signos de puntuación. Como ejemplos, valgan los siguientes:

22. Comienza con las grafías después avanza y se introducen palabras y después va hacia lo más complejo.

23. Se inicia la enseñanza partiendo de los elementos más complejos como el texto para ir descomponiéndolos en los más elementales.

En el ejemplo número veintidós falta una coma después de comienza con las grafías y sobra una coma después de va. En el ejemplo veintitrés, faltarían dos comas que delimitasen como el texto, pues es un inciso explicativo.

Otra vez nos encontramos con una gran abundancia de oraciones demasiado largas en los textos de esta asignatura. Estas oraciones demasiado largas tienen, por supuesto, unos problemas de puntuación. Pero la característica principal que salta en una primera lectura es el hecho de que el lector no puede acceder totalmente al significado de la oración, pues se pierde en su lectura, como en el caso siguiente:

24. Si tomamos como definición de competencia lingüística «el conjunto de conocimientos y saberes que posee el hablante para formar oraciones gramaticales», esto se refiere a mera competencia gramatical dejando de lado el uso real del lenguaje, la comunicación, por ello Hymes propuso ampliar el término al de competencia comunicativa y lo definió como «el conjunto de habilidades y capacidades de adecuación al contexto que debe de poseer un individuo para la comunicación oral», o dicho de otro modo, todos aquellos conocimientos y saberes 
que debe haber adquirido un individuo para poder enfrentarse a cualquier tipo de situación comunicativa concreta.

Esta oración la podríamos dividir en tres oraciones más cortas. El párrafo quedaría así:

25. Si tomamos como definición de competencia lingüística «el conjunto de conocimientos y saberes que posee el hablante para formar oraciones gramaticales», esto se refiere a mera competencia gramatical dejando de lado el uso real del lenguaje, la comunicación. Por ello Hymes propuso ampliar el término al de competencia comunicativa y lo definió como «el conjunto de habilidades y capacidades de adecuación al contexto que debe de poseer un individuo para la comunicación oral». $\mathrm{O}$ dicho de otro modo, todos aquellos conocimientos y saberes que debe haber adquirido un individuo para poder enfrentarse a cualquier tipo de situación comunicativa concreta.

Otra de las consecuencias de la realización de estas oraciones tan extremadamente largas es que muchas veces se producen errores de concordancia en el interior de ellas, pues hay una excesiva distancia entre dos elementos que deben concordar en género o en número, como por ejemplo:

26. En el segundo nivel, el niño, que ya ha empezado su etapa de iniciación verbal, ya es capaz de reconocer ciertos símbolos concretos que aparecen a su alrededor y que son comunes, pueden reconocer estos símbolos porque están habituados a ellos.

En esta oración el alumno está dando una características del niño y utiliza como sujeto de la oración el niño, en una forma singular y generalizadora. Sin embargo, a la hora de establecer la concordancia con el núcleo del predicado, escribe el verbo en plural.

A lo largo de estos textos no encontramos un uso ni muy profuso ni particularmente extraño de los marcadores del discurso. Si algo llama la atención es que el alumno no utiliza los marcadores del discurso en este tipo de textos. Se limita a ir añadiendo unos párrafos a otros.

\subsection{Errores de adecuación}

Otra de las características que también encontramos en este tipo de textos es que también aquí se emplean términos y expresiones propios del habla informal coloquial. Esto sucede en veinticinco de los casos. Valgan como ejemplos: 
27. Al principio de su desarrollo lingüístico el niño se arma un follón con determinados fonemas de la lengua.

28. Este método es mejor visto por muchos expertos en didáctica de la lengua, que lo consideran más eficaz.

En el primero de los ejemplos el alumno utiliza la expresión claramente coloquial armarse un follón. Sería mucho más correcto utilizar el verbo confundir en este contexto. En el segundo ejemplo, la expresión ser mejor visto está utilizada también de una forma incorrecta. Esta expresión coloquial, además, no es adecuada por su significado. Sería mucho más correcto utilizar es preferido.

También en estos textos se siguen utilizando palabras comodín. Treinta de los ejemplos analizados contienen algún uso de este tipo de palabras. Uno de los ejemplos sería:

29. Esta proximidad entre los fonemas hace que el niño tenga ciertas dificultades a la hora de adquirirlos.

En este caso, sería mucho más preciso utilizar el verbo provocar en lugar del verbo hacer.

\subsection{Errores de ortografía}

Una vez más, los errores más comunes relacionados con la ortografía se plantean en el nivel de la acentuación. Encontramos ausencias de tildes en treinta y ocho de los exámenes. Por ejemplo:

30. Hay distintos metodos para potenciar que el niño interactue con el maestro de forma fluida y espontanea.

31. Es mejor que el niño trabaje a partir de ejercicios que le permitan relacionarse con sus compañeros. Éstos le servirán de guia y apoyo en su evolución.

En el ejemplo número treinta se echan en falta tres tildes: métodos, interactúe y espontánea. En el ejemplo treinta y uno encontramos el caso contrario, estos no debe llevar tilde. Volvemos a encontrar un caso de confusión relacionado con las tildes diacríticas. También en este ejemplo falta una tilde en guía para romper el diptongo. 
En cuanto a errores relacionados con las grafías de las palabras, provocados o no por la interferencia lingüística con el valenciano, aparecen de forma mucho más residual que los problemas tratados hasta ahora. Únicamente en seis de los textos podemos encontrar errores relacionados con la mala escritura de alguna palabra, como el siguiente ejemplo:

32. Aprovechar lo que el alumno ya conoce de la realidad, o $a$ aprendido en el aula, para iniciar actividades.

\section{CONCLUSIÓN}

Como hemos podido observar a lo largo de los ejemplos anteriores, nuestro alumnos universitarios no poseen el nivel $\mathrm{C} 1$ de español, al menos en lo que se refiere a textos escritos. Hemos observado que muy pocos de los textos construidos por los alumnos son textos formalmente perfectos. Más allá del contenido evaluable, los textos de los alumnos fallan como textos en sí.

Hemos observado que los errores que se producen van más allá de las tradicionales faltas de ortografía, que hasta no hace mucho tiempo centraban toda la atención de los profesores. Los errores que cometen nuestros alumnos son errores más profundos y más ligados con la ordenación del texto. En primer lugar, observamos que los alumnos fallan en lo que se refiere a la ordenación general del texto. En el primer grupo de textos pertenecientes a la asignatura «Lengua española para maestros», sí que la mayoría de textos estaban construidos en base a una estructura externa reconocible. Posiblemente, esto es debido a que durante la asignatura se les insistía mucho en este hecho. El problema viene cuando los alumnos tienen que enfrentarse a otro tipo de textos, como es el caso de la segunda asignatura. En este caso, la mayoría de los alumnos no dota al texto de una estructura reconocible. Relacionado con este hecho, en el ámbito de la coherencia, observamos que tanto en la asignatura de primer año como en la asignatura de segundo año, los alumnos fallan en la construcción de párrafos. No tienen muy claro cuáles son las bases para construir párrafos ni cómo llevarlas a la práctica de forma efectiva.

Si entramos en el nivel de la coherencia, podemos constatar que estas dificultades que el alumno tiene a la hora de construir estructuras textuales y párrafos, se trasladan también a la construcción de las oraciones. El alumno tiene dificultades en el nivel oracional, pues no tiene muy claro como limitar y relacionar las distintas oraciones que conforman un párrafo entre sí. Parece que el problema radica sobretodo en que el alumno no tiene muy claro cómo usar los signos de puntuación. El uso de los signos de puntuación es básico en el nivel oracional. Es el elemento relacionante básico entre las distintas oraciones. A esto también se une el hecho de que existan problemas en el uso 
de conectores y marcadores del discurso. Estos dos hechos hacen que el alumno tenga problemas para organizar la información a nivel oracional y para relacionar las distintas oraciones entre sí. Como resultado, el alumno realiza construcciones oracionales demasiado largas, pues al no saber relacionar la información de forma adecuada, engloba demasiada de ella dentro de una misma oración.

En cuanto a los errores relacionados con la adecucación, el error más significativo es el uso de un lenguaje no apropiado en este tipo de textos. Cada vez más en el ámbito de la educación se va perdiendo el ámbito de la formalidad. Por eso, los alumnos muchas veces tienen problemas para poder expresarse, tanto de forma oral como escrita, en una variante formal sin interferencias de usos informales.

Finalmente, los errores ortográficos siguen apareciendo en este tipo de textos. Sin embargo, la frecuencia de aparición de este tipo de errores es mucho menor que en los demás casos. Posiblemente sea porque desde los primeros estadios educativos, se les insiste mucho a los estudiantes contra este tipo de error.

A la vista de estos resultados se nos plantean una serie de cuestiones. La primera de ellas: ¿Realmente los alumnos que ingresan en la universidad tienen un nivel de español suficiente para poder asegurar que cuando acaben su periplo universitario saldrán con un nive $\mathrm{C} 1$ de español? Creemos que la respuesta a esta pregunta es no. Los alumnos poseen grandes dificultades a la hora de escribir textos. Y, en nuestra opinión, no son dificultades por desconocimiento de reglas gramaticales o desconocimiento de reglas ortográficas. Los alumnos tienen problemas en la forma de aplicar esas reglas para trasladarlas a un producto concreto como puede ser un texto. Además, entre los textos producidos en el examen de primer año y los textos producidos en los exámenes de segundo año, no se observan diferencias significativas, se siguen cometiendo los mismos errores o muy parecidos.

La segunda de las cuestiones es: ¿Realmente los planes de estudio de los diferentes grados garantizan que el alumno pueda alcanzar ese nivel a lo largo de su periplo universitario? Hasta donde nosotros conocemos la respuesta es no. Basándonos en nuestra experiencia como profesores de los grados de magisterio, los nuevos planes de estudio únicamente cuentan con una asignatura dedicada a la enseñanza del español como sistema, «Lengua española para maestros». El problema es que es, a todas luces insuficiente, ya que, con una sola asignatura cuatrimestral, no se pueden solucionar todos estos problemas que los alumnos tienen a la hora de enfrentarse con la producción de textos. En otros planes de estudio, hasta donde conocemos, la situación no mejora ostensiblemente. $\mathrm{O}$ hay una asignatura dedicada a la enseñanza del español como sistema o no hay ninguna asignatura que se ocupe específicamente de este asunto.

Estas dos cuestiones nos llevan finalmente a una tercera, que es la más importante: nosotros, como profesores, ¿qué debemos, o qué podemos hacer frente a esta situación? La verdad es que esta pregunta tiene difícil respuesta y aún más difícil aplicación. Lo único que parece claro es que no podemos dejar de lado esta cuestión y que debemos plantearnos que si no hacemos nada a este respecto, nuestros alumnos pueden tener dificultades bastante grandes a la hora de construir textos en la vida real. 
REFERENCIAS BIBLIOGRÁFICAS

Aleza IZQuiERDO, Milagros, coord. ${ }^{a}$ (2010): Normas y usos correctos en el español actual, Valencia, Tirant lo Blanch.

Bosque, Ignacio y Violeta Demonte, eds. (1999): Gramática descriptiva de la lengua española, Madrid, Espasa Calpe.

CASSANY, Daniel (2000): Construir la escritura, Barcelona, Paidós.

CASSANy, Daniel (2005): Describir el escribir: cómo se aprende a escribir, Barcelona, Paidós, 13. a edición.

CASSANY, Daniel (1993): Reparar la escritura, Barcelona, Paidós.

CASSANY, Daniel (2002): La cocina de la escritura, Barcelona, Anagrama.

DiCCIONARIO DE PARTíCUlAS DISCURSIVAS DEL ESPAÑOL. DPDE. [en línea]: http://textodigital.com/P/DDPD/ [consulta: 20/08/2011].

GóMEZ TORREGO, Leonardo (2005): Nuevo manual del español correcto. Madrid, Arco Libro.

GómEZ TORREGO, Leonardo (2011): Las normas académicas: últimos cambios, Madrid, SM.

Montolío, Estrella, coord. ${ }^{\text {(2000): }}$ Manual práctico de escritura académica, Barcelona, Ariel, Vols I y II.

REAL ACADEMIA ESPAÑOla Y AsOciación DE ACADEMIAS DE LA LENGUA EsPAÑOLA (2005): Diccionario panhispánico de dudas, Madrid, Santillana.

REAL ACADEMIA EsPaÑola Y ASOCIACión DE ACADEMias DE LA LENGUA ESPAÑOla (2009): Nueva Gramática de la Lengua Española, Madrid, Espasa.

Real ACAdEMia EsPañola y Asociación de ACAdEMias de la Lengua ESPAÑOLA (2010): Ortografía de la lengua española, Madrid, Espasa.

REYES, Graciela (1998): Cómo escribir bien en español. Manual de redacción. Madrid, Arco Libros. 
